(Amsterdam), B. Giraud (Saclay), D. Ponting (Glasgow) and Z. Szymanski (Warsaw) went deeply into a few of those theoretical problems whose solutions owe a lot, if not all, to the existence of large computers.

On the more technical side, the very important role which computers and computational methods play in experiments with large amounts of data, came across very clearly in the talks on "Trigger and Decision Processors" and "Influence of Computational Algorithms on Experimental Design". They showed that a good fraction of the experimenters' ingenuity goes into the clever use of digital processors at all stages of data reduction.

Finally, several talks dealt with recent developments of hardware and general software. Central computing facilities, mass storage, on-line systems, programming languages and libraries, large scale data transmission over long distances, data bases in physics and algebraic computation were discussed and speculations made about their future.

The contributed papers were grouped into four half-day sessions. The most popular subject turned out to be "On-line computing", which provided a forum for those numerous and impressive projects where computers, mostly in the form of programmable or microprocessors, have been brought into the data acquisition electronics. In the other sessions headed "theory", "data processing" and "graphics", many lively and interesting exchanges between specialists took place without perhaps showing trends as clear as the shift towards decentralisation emerging from the "on-line" session.

\section{Personal Impressions}

My personal reactions to the four day meeting are as follows. First, there is this clear fact: Large scale integration has brought the performance and price of processing units and memories within reach for a multitude of applications, which gladly use such decentralised intelligence for systems of higher performance and flexibility. Algorithmic techniques are thus entering a domain where previously, simple Boolean logic in hard-wired form, was the end. It is probably safe to say that this development is only at its beginning; the hard-ware market is still evolving fast, and many non-physics applications on a large scale (and of commercial interest) are experiencing the same evolution.

Second, this decentralisation does not seem to have the effect expected by some, of making the familiar large multi-user systems in our computer centres look obsolete. On the contrary, demands on central facilities seem to be growing as ever, which can perhaps be explained by the higher quality output which the more sophisticated systems produce under the influence of added decentralised capacity. To be sure, the type of demand on central facilities does change. There is much demand for storage capacity, fast and reliable peripherals, services like filing schemes, editing and graphics facilities - and, of course powerful and reliable communication between the computers inside a laboratory and from lab to lab.

Last, some well-known problems are still with us. Like a pedal point on the organ, there was a common undertone in many talks, culminating in a plea for collaboration on standards (issued on behalf of a subcommittee of the European Committee for Future Accelerators). The effort to tame all the available systems and to get at the real problem is estimated by many to be larger than necessary, because the physics computing community is not sufficiently organised itself, and not sufficiently guided from outside in anything going beyond the most basic operations. A multitude of solutions is frequently offered for a given technical problem, for which, at best some local guidance is offered. More typically, the user is left to choose himself, a choice he does not want to spend time on. Sometimes, the "suppliers" of solutions even are in open competition. It may well be that the size of the physics and science community has something to do with this. A smaller sized group finds naturally its style of collaborating, and a large community makes the problem so apparent that solutions are

\section{HADRONIC PRESS}

Nonantum, Massachusetts 02195

announces the yearly series of

reprint volumes

\section{Developments in the Quark Theory of Hadrons}

\section{Edited by Professors \\ DON B. LICHTENBERG and \\ S. PETER ROSEN}

Volume 1 (1980), U.S. \$50. inclusive of handling and surface mail to all countries. Optional air freight: U.S. \$7. for Europe and U.S. \$10. for Asia.

The first volume presents a selection of papers, both theoretical and experimental, that take the reader from the invention of quarks to their more recent developments in hadron physics, up to December 31, 1978.

The series will then continue with a yearly volume identifying the most relevant advancements in the field. Each volume contains an introduction by the Editors as well as suggestions for further reading material.

enforced. As one participant put it: I wish, the night was over.

The proceedings of this conference will be available later as a special issue of Computer Physics Communications. The abstracts are now available as Volume $4 \mathrm{G}$ of the Europhysics Conference Abstracts Series.

\section{R. Bock, CERN}

\title{
ESSDERC '80
}

This year it was the United Kingdom's turn to be host to ESSDERC which was held at York University from 15-18 September. Ten years of solid state device research have seen immense changes in the devices and their associated technology. Large scale integration and now, very large scale integration, are exceedingly demanding on reliability, reproducibility, packing density and power dissipation to name but a few of the problems. This emphasis on technology is reflected by the integration of the Tenth European Solid State Device Conference with the Fifth Symposium on Solid State Device Technology. The importance of technology was emphasised by B.O. Kolbesen and $H$. Strunk from Siemens, Munich, where Kolbesen had collected a beautiful set of slides illustrating swirl effects and other "Process-Induced Defects in Silicon". The correlations he established between various defects and device failures were impressive. Fortunately, the invited talks in both the symposium and the "main" device conference were joint sessions and there surely must be a case for scrapping the slightly artificial division between the two events which are, in any case, joint events next year. Devices need technology and technology needs devices.

It is appropriate at a research conference to consider the future, and the possible devices of the future are again dominated by technology, especially dominated by the questions relating to the limits to size. Device physics will change drastically if we reduce the size of the devices down to the quantum scale of nano-metres. However, E. Constant of CRNS, Lille, in reviewing this field, wisely limited his main discussions to devices where macroscopic quantities such as momentum, energy, and relaxation times still have some meaning. Monte-Carlo calculations are acknowledged as perhaps the most powerful method of attack, and Constant gave a few examples, which were backed up in later papers, for example, one by C. Moglestue from Reading University on a simulation of 
a field effect transistor with a $0.1 \mu \mathrm{m}$ gate. The inability of the electrons to be scattered by phonons when dealing with picosecond time scales makes the electrons travel faster - ballistic regime - and can give most impressive maximum operating frequencies, perhaps beyond $100 \mathrm{GHz}$.

In looking to the new future of submicron devices, materials growth particularly in the compound semiconductors, will play a key role. This topic was reviewed by R.W. Brander and M.M. Faktor of the British Telecommunications Research Laboratories. Brander clearly came down, in the presentation, on the side of expecting molecular beam epitaxy to give the best technique for controlled and selective material growth, at least in the future. The problems of throughput that may trouble mass production were not emphasised by Brander, but were of concern to P.J. Daniel (Philips' Research Laboratories, Redhill) in his careful comparison of photolithography and electron-beam lithography. While conceding that the electron beam has clear advantages over photo-lithography in the diffraction limitations, in spite of back scattering of electrons blurring the edges, Daniel believed that the problems of speed of writing for raster or vector scanned e-beam machines would remain. If true, this could have serious consequences on the commercial exploitation of the new physics of sub-micron devices where e-beams may be essential rather than as at present, used to help in discretionary wiring of VLSI.

Indeed the practical difficulties of reducing dimensional tolerances to increase packing densities while maintaining yield in VLSI production processes, may have accounted in part, for the greater interest in vertical integration which appeared in papers time and again. For example, J. Lohstroh (Philips' Research Laboratories, Eindhoven) in his review paper indicated how vertical integration was helping the packing density of bipolar devices and also reducing the power consumption to meet the requirements of VLSI, even with $3 \mu \mathrm{m}$ minimum detail. Vertical integration appeared again in the opening review paper of the conference from J. Tihanyi (Siemens, Munich). Tihanyi showed how the VMOS, which originally had the V standing for the shape of its special groove, now had the $V$ standing for vertical. Vertical integration had given Siemens a process whereby MOS power devices could be made with reverse hold off voltages of around $1000 \mathrm{~V}$, with on-conductances of over 100 Siemens (to quote Tihanyi's pun). Bernd Hoefflinger of Dortmund University again echoed some of these ideas about vertical integration in discussing enthusiastically the virtues of CMOS. On listening to Hoefflinger's technology which combines bipolar and MOS in an appropriate blend of planar and vertical integration, one is led to believe that CMOS will give the ultimate in flexibility of design from high speed, low power, through to high voltage, if required; an exciting prospect for VLSI.

Special mention must be in order for the contribution of Cyril Hilsum, (Royal Signals and Radar Establishment, Malvern). Hilsum was described by Walter Merz as the "father" of ESSDERC, having played an important role in forming ESSDERC ten years ago and guiding the conference through all the intervening years. In a relaxed mood before the conference outing to the Yorkshire Moors, Chatsworth House or the beautiful York City, Hilsum gave a colourful discourse on solid state displays. $\mathrm{He}$ showed, for example, a unique photograph of a $512 \times 512$ matrix CdS display from the Kirin Inst. of Physics, Chang Chun, but pointed out that it emitted only $1 \mathrm{ft}$ lambert, some 30 times less bright than a television set. However, the future was brighter than that and liquid crystals had made a dramatic advance in reliability with the production of improved materials. Improved materials would again lead to colour being added to liquid displays without the rapid bleaching of some of the present methods. The next ten years he promised would not be dull.

To a man who is interested in the physics of novel devices, one likes to think that ESSDERC will report on them. The invention of such novel devices is slowing down but it was a pleasure to hear that $\mathrm{J}$.
Shannon's (Redhill) Monolithic Hot Electron Transistor, which was first reported in the IEE Journal on Solid State and Electron Devices, was continuing to do well. Its "camel" diode emitter-base indicated satisfactory transport of the hot electrons and gave promise for future high microwave developments. G.G. Bloodworth's (York) fascinating rotating domain magnetometer continues to provide interest and the explanation of the physics of his device is now much clearer.

ESSDERC then is very much alive as nearly 300 delegates, from 25 countries, know. Like Hilsum's promise for displays, the next ten years will not be dull.

Next year's ESSDERC will be held from September 14-18 at Toulouse and again the Symposium on Technology will be held in parallel. General information for ESSDERC ' 81 may be obtained from:

\section{Dr. G. Sarrabayrouse,}

Laboratoire d'Automatique et d'Analyse des Systèmes - C.N.R.S.,

7. Avenue du Colonel Roche,

31400 Toulouse.

Tel. (61) 25.21.47

Telex: LAASTSE $520930 \mathrm{~F}$

Abstracts of the papers presented to ESSDERC ' 80 are published in the Europhysics Conference Abstracts Series, Volume $4 \mathrm{H}$.

\section{J.E. Carroll, Cambridge}

\section{ESCAMPIG}

The Vth European Conference on Atomic and Molecular Physics of lonized Gases, ESCAMPIG was held in Dubrovnik 1-3 September. This conference is now well established and the organizers, R. Deloche and R.K. Janev, chose to include several new topics related to the same central theme. This year, for the first time the collisional properties of Rydberg states ( $F$. Gounand) and laser induced inelastic collisions (C. Manus) were presented.

Due to their large size and the weak binding of the outer electron, Rydberg atoms are very sensitive to external perturbation. Gounand gave a very enlightning survey of the collisional properties of these very high atomic excited states, underlining the importance of their role in astrophysics and in laser isotope separation.

Currently the most widely studied process is the total collisional de-excitation (quenching) of a well-defined Rydberg level. Two-step selective excitation of alkali states, for example, is reached by pulsed dye lasers. Large depopulation crosssections are generally observed $\left(10^{-13}-\right.$ $10^{-12} \mathrm{~cm}^{2}$ ). The most interesting behaviour is exhibited by the $n$ dependance. At rather small values of $n$, the cross section increases with $n$, reaches a maximum, then decreases for still higher $n$ values. It was also shown that the position of the maximum shifts to higher $n$ values when the mass of the perturber increases. These findings of the Saclay and S.R.I. groups seem to emphasize the dominant role of the electron perturber interaction. although in other situations the influence of the ionic core may be more important.

Due to the importance of the subject, this talk was followed by a workshop opened by Deloche, who presented a general account of the various reactions that involve Rydberg states, such as the ionizing processes observed by R.F. Stebbings and A.N. Klutcharev. Deloche also discussed the present situation of the theoretical models and their ability to describe the experimental findings.

The growing importance of laser isotope separation has been very often emphasized. N.V. Karlov presented a very clear survey of the basis and possibilities of such a field. For a great number of atoms, an enrichment of a factor of 100 or even higher has already been obtained. A selective first step laser excitation carries the 